\title{
Determining the mass-related reaction effectiveness factor of large, nonspherical fuel particles for bridging between intrinsic and apparent combustion kinetics
}

\author{
Tibor Szűcs $^{1}{ }^{\mathbb{D}} \cdot$ Pal Szentannai ${ }^{1}$
}

Received: 17 December 2018 / Accepted: 20 November 2019 / Published online: 30 November 2019

(c) The Author(s) 2019

\begin{abstract}
The utilization of challenging solid fuels in the energy industry is urged by environmental requirements. The combustion kinetics of these fuel particles differs markedly from that of pulverized coal, mainly because of their larger sizes, irregular (nonspherical) shapes, and versatile internal pore structures. Although the intrinsic reaction kinetic measurements on very small amounts of finely ground samples of these particles are mostly available, a bridge toward their apparent reaction modeling is not evident. In this study, a method is introduced to build this bridge, the goodness of which was proved on the example of an industrially relevant biofuel. To do this, the results of a macroscopic combustion measurement with real samples in a well-modelable environment have to be used, and for considering some not negligible effects, 3D CFD modeling of the experimental environment is also to be applied. The outcome is the mass-related reaction effectiveness factor as a function of the rate of conversion. This variable can be considered as the active fraction of the entire particle mass on its periphery, and it can be used as the crucial element in modeling the combustion process of the same particle under other circumstances by including the actual boundary conditions. Another advantage of this method is its covering inherently the entire combustion process (water and volatile release, and char combustion) and also its applicability for reactors utilizing bigger particles like fluidized bed combustors.
\end{abstract}

Keywords Nonspherical solid particle $\cdot$ Macro-sized particle $\cdot$ Combustion kinetics $\cdot$ Waste-derived fuel · Biomass

$\begin{array}{ll}\text { List of symbols } \\ A & \text { Pre-exponential factor }\left(\mathrm{s}^{-1}\right) \\ A_{\mathrm{m}} & \text { Mass-related specific internal surface area }\left(\mathrm{m}^{2} \mathrm{~kg}^{-1}\right) \\ c & \text { Mass ratio } \\ c_{\mathrm{ox}} & \text { Oxygen concentration in the bulk phase }\left(\mathrm{mol} \mathrm{mol}^{-1}\right) \\ E & \text { Activation energy }\left(\mathrm{J} \mathrm{mol}^{-1}\right) \\ k_{0} & \text { Kinetic coefficient }\left(\mathrm{s}^{-1}\right) \\ k_{\mathrm{A}} & \text { Kinetic coefficient related to surface }\left(1 / \mathrm{m}^{2} / \mathrm{s}\right) \\ k_{\mathrm{d}} & \text { Mass transport rate (intrinsic) }\left(\mathrm{s}^{-1}\right) \\ k_{\mathrm{r}} & \text { Kinetic reaction rate (intrinsic) }\left(\mathrm{s}^{-1}\right) \\ m & \text { Kinetic parameter } \\ m & \text { Sample mass }(\mathrm{kg}) \\ n & \text { Kinetic parameter }\end{array}$

Tibor Szúcs

szucs@energia.bme.hu

Pal Szentannai

szentannai@energia.bme.hu
$R \quad$ Universal gas coefficient $\left(\mathrm{J} \mathrm{mol}^{-1} \mathrm{~K}^{-1}\right)$

Sh Sherwood number

$t \quad$ Time (s)

$T \quad$ Temperature (K)

$x \quad$ Conversion rate

$z \quad$ Kinetic parameter

$\eta \quad$ (Surface-related) reaction effectiveness factor

$\eta_{\mathrm{m}} \quad$ Mass-related reaction effectiveness factor

\section{Subscripts and superscripts}

app Apparent

int Intrinsic

A Ash

M Moisture

calc Calculated

meas Measured

ox Oxygen

V Volatile

0 Initial

1 Department of Energy Engineering, Faculty of Mechanical Engineering, BME - Budapest University of Technology and Economics, Muegyetem rkp 9, Budapest 1111, Hungary 


\section{Introduction}

Biomass combustion is widely considered as one of the main pillars of renewable and sustainable energy of the future. One of the most investigated topics is the prediction of heat release, which needs a proper particle combustion model. The full process of combustion consists of three main parts: drying, devolatilization, and char combustion [1]. The description of these processes is highly related to the size and the shape of the particles. Usually, for small and spherical samples, a simpler method is enough, but the bigger and highly nonspherical ones call for a more complex model.

The researches carried out in this field of combustion kinetics could be categorized into two main groups, intrinsic and apparent kinetics [2,3]. Intrinsic kinetics is purely related to the chemical aspect of the process neglecting every other external phenomenon, while apparent kinetics aims to describe the combustion of a realistic particle with every influential parameter as well. While these two processes are obviously connected, their main ideas are different. That is why, albeit apparent kinetics usually consists of reaction kinetic parameters, it could not be converted directly from the intrinsic kinetics of the sample [3].

Two of the most recent studies $[1,4]$ reviewing the topic summarized the available technics with a highlight on specific applications (fixed and fluidized beds). It was stated that a proper model for big, nonspherical particles does not exist yet.

Drying of the sample is very often excluded from the investigations; however, it can be considered as a part of the volatile release procedure owing to the very similar character of its basic nature and discussion [1]. Also, this approach is followed in the present report.

The devolatilization of different biomass fuels is widely researched, as it is the key process not only for combustion, but also for the pyrolysis and gasification applications. The field was reviewed thoroughly by Gomez-Barea and Leckner [3] and Di Blasi [5]. For devolatilization, in case of small particles, intrinsic kinetics was identified as the main controlling process. However, with bigger ones the process shifts to heat transfer, due to the time it takes to heat up all parts of the sample to the temperature, that the reaction would require. To categorize fuels according to this, the $P y$ number was developed as the dimensionless ratio of heat conduction and intrinsic kinetics of the sample [4].

Regarding char combustion, there are numerous methods to describe the combustion of relatively small and spherical particles. The first one is from the 1960s $[6,7]$, widely used even nowadays mostly for coals, as it is quite precise with only geometrical limitations. The basics of the mode concerned are as follows. The combustion is controlled by the reaction kinetics and the oxygen transport (the latter both in the gas near the sample and in the ash). The importance of the particle shape and its modifications was also considered in the form of a dynamically changing reaction surface which was determined through complex measurements.

The theoretical background was established by Levenspiel [8], who developed mathematical models for spherical particles for different combustion cases based on which controlling process is the dominant one, and how the particle changes during the conversion. In a progressive-conversion model (PCM), the reaction takes place homogeneously in the whole volume of the sample, while in a shrinking-core model (SCM) only the surface reacts. Generally, the PCM is suitable for small particles, while the SCM is able to describe bigger ones as well.

To determine the intrinsic kinetics of a sample, the most common technics are the TG measurements. Regarding this method, it is important to point out that the measurement always takes place in the kinetic regime (Regime I as per [2]), and the external influencing factors (i.e., heat transfer) are oppressed. Numerous investigations were made for the kinetics of biomass fuels, that is reviewed by Di Blasi [5, 9], and more recently by Wang and coauthors [10]. The usually used form for describing the conversion of the sample is as follows [9]:

$\frac{\mathrm{d} x_{\mathrm{int}, 1}}{\mathrm{~d} t}=A \mathrm{e}^{-\mathrm{E} /(\mathrm{RT})} f(x)$,

where 1 in the subscript distinguishes the current one-component case from more complex particles to be discussed later.

Many functions are available for $f(x)$ (well summarized by Aboulkas et al. [11]) to describe the surface reactivity change due to structural changes of the sample [12]. The most frequently used one is in the form of order equations. An enhanced version of it is also developed and successfully tested for various biomass samples as follows $[10,12]$ :

$\frac{\mathrm{d} x_{\text {int }, 1}}{\mathrm{~d} t}=A \mathrm{e}^{-\mathrm{E} /(\mathrm{RT})}(1-x)^{\mathrm{n}}(x+z)^{\mathrm{m}}$.

In case of complex samples (most wastes and biomass), the conversion usually could not be described by one equation, so the common method is to divide it into different fractions with different conversion equations, and the conversion of the whole sample is calculated by the sum of them $[13,14]$. This technique was also successfully used in industrial-scale CFD models [15].

The already mentioned problem regarding the nonspherical particles has become an even more frequently investigated topic in the past few years. There are a lot of studies 
investigating the modifying effect of the shape irregularities, but most of them still examine small (few millimeters) particles in laboratory environment, with a few exceptions. Most of them concern only the conversion of char by neglecting the volatile release, but as the main problems and the applied methods are similar, all of them are handled together.

Moneni and coworkers [16] identified the problem of the real-sized and real-shaped particles and made measurements (with ca. $3 \mathrm{~mm}$ samples) to describe them. The importance of the oxygen mass transport and the different shapes were pointed out by measuring the burn-out time of biomass samples with different geometries. It was found that the mass loss is much faster in case of cylindrical particles than spherical ones, but no combustion model was developed. Bibrzycki et al. [17] also performed measurements and developed a model for realistic char samples, with the diameter of 5.2-6.3 $\mathrm{mm}$. In the aforementioned work, a connection between the intrinsic and the apparent kinetics of the sample was made by measuring the mass change in preheated gas, and the results were fitted into a shrinking-core model. In this way, the internal surface area development of the sample could be derived.

The effect of the shapes during the combustion of relatively small $(200 \mu \mathrm{m})$ fuel chars was examined theoretically and experimentally by Schiemann et al. [18]. They calculated the reaction rate with a typical energy balance equation by the assumption of different base fuel geometries. The observed deviation was small, but still relevant.

The geometry dependency of the reactivity of chars was investigated thoroughly by Liu and coworkers [19], and an intrinsic model was also developed. As mainly the problems of the TG and DSC measurements were investigated, the applied samples in this work are relatively small (few mg-s). However, the used method to describe the specific surface activation rate changes during the conversion could be a great starting point to describe bigger samples as well. With this method, the application of the classical idea of Field [6, 7], namely to compensate the drawbacks of the TG and DSC measurements, was also presented.

In a study of Pereira and Pinho [20], the combustion behavior of several biomass chars with a diameter of a few millimeters was investigated, by emphasizing not only the effects of the nonsphericity but also of the fragmentation. Measurements were taken in a fluidized bed reactor, and the results were evaluated by a reaction model. It was found that ignoring the fragmentation means an error of 5 and $10 \%$ in the diffusive and kinetic data, while ignoring the shape of the particles means a more relevant, 45 and $50 \%$ error, respectively.

Johansen et al. [21] developed a model to describe specifically the fast devolatilization of woody biomass fuels. A laboratory-scale experiment in a laminar flow reactor was used to derive the kinetics, which was implemented into a
CFD model of a bench-scale equipment to iterate the exact temperature profile of the fuel particles in those conditions. The diameter of the particles was low (around $0.1 \mathrm{~mm}$ ) and their shape was not handled at all, but the connecting method between the laboratory-scale and the bench-scale results through a CFD model is worth mentioning.

The mass transport around fuel particles in realistic conditions was investigated thoroughly. The most recent overview is made by Scala [22]. His work includes a detailed comparison of the available Sherwood number formulas, with an emphasis on the cases related to fluidized beds. However, regarding the nonspherical big particles, only the absence of a correct description was mentioned, the commonly used way is to use a sphere-like description and modify it as necessary. So, it is clear that this field also lacks a proper method to handle these particles. Li and Zhang [23] made a theoretical study on the combustion of ellipsoidal char particles in the diffusive regime, pointing out the relevance of the shape. In case of particles with a more different shape, this effect could be more relevant and harder to describe with purely analytical models.

The key contribution of the current work is setting up and testing a technique for supplying the apparent kinetic model of the entire combustion process of large, nonspherical particles on the basis of intrinsic reaction kinetic data. The resulted model inherently covers the entire combustion process including drying, devolatilization, and char combustion. It is, therefore, directly applicable for reactor design and control of actually very relevant, problematic fuels such as biomasses and waste-derived fuels. The technique itself is composed of some experimental and numerical steps to be demonstrated through an example of a selected biofuel in the next section. The main output of the method is the newly created mass-related reaction effectiveness factor, which represents all parameters controlling the combustion process as a function of the sample's conversion. This function was determined for the selected prototype sample, the sunflower seed shell pellet.

\section{Materials and methods}

Bridging between the intrinsic kinetic information (e.g., TG diagrams) and the apparent combustion kinetic model of the same fuel is not evident in case of big particles. The problematic difference is that while the intrinsic description considers the entire mass of the sample to participate in the reaction, in the apparent case a central, hidden portion of a big particle is obviously inactive. Let us consider a particle of any size and shape, and let us consider its virtual fraction on its periphery, supposing that its entire mass participates in the reaction with the gas molecules reaching the surface! With introducing the variable $\eta_{\mathrm{m}}$ to represent this mass ratio, 
this variable is intended to be used as the bridge between the intrinsic and apparent descriptions.

Note that the external, active mass $\eta_{\mathrm{m}} \cdot m$ on the periphery of the entire particle mass $m$ is not considered to behave just like the sample in the TG apparatus or in any other intrinsic measuring devices. It is considered to behave as the subsequent samples would behave if the gas concentration around them would be the same as the concentration directly around the surface of the big particle. Also note that $\eta_{\mathrm{m}}$ can be considered in many cases as a consequence of the internal pore structure of the fuel particle, that is, the mass-related specific internal surface area $A_{\mathrm{m}}$ and its effectiveness factor $\eta$ representing the degree of gaseous penetration $[2,3]$. From another point of view, $\eta_{\mathrm{m}}$ can also be considered as a consequence of a certain reaction depth of the solid fuel particle. However, characterizing the pore structure is rather difficult in many cases concerned due to complicated measuring techniques developed mainly for coal, extraordinarily high volatile content resulting in big and varying pore structure, and irregular particle geometry and density. Similarly, the concept of reaction depth could be well applied for particles of well-defined geometry, but this is definitely not the case for waste-derived fuels. Further, because the shapes of most particles of this type are rather flat (almost 2D, [24]), the combustion takes place much more actively on the edges. All in all, instead of trying to find deeper physical understanding of $\eta_{\mathrm{m}}$, its general and overall meaning will be considered throughout this paper, as introduced above.

Let us name $\eta_{\mathrm{m}}$ as mass-related reaction effectiveness factor, appearing to be a key element in bridging between intrinsic and apparent reaction kinetics of big, irregularshaped solid fuel particles. The task is, then, to find this value, or more generally, to develop a method for determining it for any specific particle. Because of investigating all steps of the combustion process, and because intensive and significant volatile release takes place throughout this process, this variable must be handled and calculated as a function of the overall (not local!) conversion rate defined as

$x=\frac{m_{0}-m}{m_{0}-m_{\mathrm{f}}}$,

where $m_{0}$ and $m_{\mathrm{f}}$ are the initial and final total masses of the sample prior to and after the entire conversion process, respectively, and $m$ is its actual value.

On this basis, the apparent combustion kinetic model of a particle with very low ash content can be written as

$\frac{\mathrm{d} x_{\mathrm{app}}}{\mathrm{d} t}=\left\{\begin{array}{ll}\eta_{\mathrm{m}} k_{\mathrm{r}} & \text { if } x \leq X_{\mathrm{M}}+X_{\mathrm{V}} \\ c_{\mathrm{ox}}\left(\frac{1}{\eta_{\mathrm{m}} k_{\mathrm{r}}}+\frac{1}{k_{\mathrm{d}}}\right)^{-1} & \text { if } x>X_{\mathrm{M}}+X_{\mathrm{V}}\end{array}\right.$,

the variables of which are summarized in Table 1 . This table gives an overview about the method of determining the variables throughout the modeling period with references to upcoming sections of this paper. Further, the last column of the same table summarizes how to use these variables when applying the model in actual specific calculations.

Note that although the two parts of Eq. (4) are similar, they cannot be combined into one simple expression because $c_{\mathrm{ox}}$ does definitely influence the char combustion procedure but the volatile release does not. And similarly, this process is not influenced by the external gas diffusion rate represented by $k_{\mathrm{d}}$. Also note that in spite of the obvious differences between the two parts, $\eta_{\mathrm{m}}$ and $k_{\mathrm{r}}$ are handled uniformly throughout the entire combustion process.

As $\eta_{\mathrm{m}}$ describes the phenomenon that not the entire mass of a big particle participates in the reaction, its value is supposed to depend upon a measure of how big the particle is compared to its initial size. Thus, the actual value of this variable must change as a function of $x$. This dependency is assumed to vary from zero to unity along with the progress of the combustion process. Any function of this character could be chosen as the basis for parameter fitting. For

Table 1 Variables of Eq. (4) and the methods to determine them throughout model development and use

\begin{tabular}{|c|c|c|}
\hline & Source while setting up the model & Source while using the model \\
\hline$k_{\mathrm{r}}(x, T)$ & $\begin{array}{l}\text { TG (intrinsic) measurement; see "Biofuel particles and their } \\
\text { intrinsic kinetic characterization" section }\end{array}$ & $\rightarrow$ From the model \\
\hline$X_{\mathrm{M}}, X_{\mathrm{V}}$ & $\begin{array}{l}\text { From the same TG measurement; see "Biofuel particles and their } \\
\text { intrinsic kinetic characterization" section }\end{array}$ & $\rightarrow$ From the model \\
\hline $\mathrm{d} x_{\text {app }} / \mathrm{d} t$ & $\begin{array}{l}\text { From macroscopic (apparent) measurement; see "Planning and } \\
\text { carrying out the macroscopic measurement" section }\end{array}$ & This is the final output of using the model \\
\hline$k_{\mathrm{d}}(x, T$, Bound.Conditions $)$ & $\begin{array}{l}\text { CFD modeling of the apparent experiment; see "Calculating the } \\
\text { particle temperature profile" section and "Calculating the gas } \\
\text { mass transfer around the particle" section }\end{array}$ & $\begin{array}{l}\text { Must be calculated (as traditionally), based on } \\
\text { the actual conditions }\end{array}$ \\
\hline$c_{\mathrm{ox}}$ & Must be given according to the experimental conditions & Must be given according to the actual conditions \\
\hline$\eta_{\mathrm{m}}(x)$ & $\begin{array}{l}\text { To be calculated based on all known variables listed above of Eq. } \\
\text { (4). This is the final result of the model development }\end{array}$ & $\rightarrow$ From the model \\
\hline
\end{tabular}


simplicity, thus, general applicability, the next form was chosen:

$\frac{\mathrm{d} \eta_{\mathrm{m}}}{\mathrm{d} x}=A_{\eta} \mathrm{e}^{-\mathrm{E}_{\eta} / \mathrm{x}}\left(1-\eta_{\mathrm{m}}\right)\left(\eta_{\mathrm{m}}+\varepsilon\right)^{\mathrm{m}_{\eta}}$

with the initial condition of

$\eta_{\mathrm{m}}(0)=\eta_{\mathrm{m}, 0}$

Finding the free parameters $A_{\eta}, E_{\eta}, \varepsilon, m_{\eta}$, and $\eta_{\mathrm{m}, 0}$ of this function is the task to do. Any available numerical optimization methods can be used.

Note that (4)-(6) is only one possible form for describing $\eta_{\mathrm{m}}$. We propose this mathematical form for several reasons. It is, namely, a rather simple, still universal function, which allows high flexibility in changing the shape, limits, and fastness of the transfer region, making it possible to be applied for a big variety of fuels.

Equations (4)-(6) summarize the form of the combustion model required for reactor simulation and design; thus, supplying its variables is the task to be carried out. This must be based on the description of the intrinsic behavior of the material (see also Table 1.), but further, it must be bridged toward the apparent behavior. The key element in this bridging is $\eta_{\mathrm{m}}$. To determine it, macroscopic (apparent) experiment must be carried out. However, the result of this experiment is determined not only by the properties of the material itself, but also by the material transport phenomenon influenced significantly also by the external boundary conditions around the particle. Eliminating this external effect would be an obvious but very difficult and maybe questionable route, and it would require a rather sophisticated measuring setup. Another route was followed in the present work, namely not eliminating, but calculating this effect. For this, a rather simple experimental setup has to be built, in which the phenomenon concerned can be considered by well-proven CFD tools. The entire procedure is discussed in the subsequent sections on the example of one selected biofuel.

\section{Biofuel particles and their intrinsic kinetic characterization}

Sunflower seed shell pellets were used as test materials originating from a Hungarian source as a commercial product. The cylindrical particles have the length of about $20 \mathrm{~mm}$ and a diameter of $6 \mathrm{~mm}$, weighting about $1 \mathrm{~g}$ each. For getting all the basic information to the actual method, TG analysis was carried out on the fuel. Although detailed analytical investigations generally apply several measure runs with different temperature slopes in most cases, for simplicity, we applied, tested, and proposed only one measurement as part of the current method. A TA Instruments SDT 2960 simultaneous TG/DTA device was used for the thermal analysis in air atmosphere $\left(130 \mathrm{~mL} \mathrm{~min}^{-1}\right)$ as described more detailed by Bakos and coworkers [25]. The pellets were ground, and a representative sample of $2 \mathrm{mg}$ was selected. The measurement was taken at $10^{\circ} \mathrm{C} \mathrm{min}^{-1}$. The resulted diagram is shown in Fig. 1 for the actual fuel, together with the indication of some numerical values to be gained from the same measurement, and which are summarized in Table 2 for the actual case.

It is important to emphasize here that TG measurements were taken on very small amount of finely ground powder of the investigated fuel, so that the intrinsic kinetics in Regime I could be assured [2, 12]. Evidently, these data cannot be applied directly for the entire mass of a bigger particle being converted definitely in another Regime.

Because of the complexity of the biofuel sample, it is handled as a mixture of several components, together resulting in the measured TG plot. This means a superposition of several terms in the general form of the intrinsic reaction rate coefficient. Additionally, the basic Arrhenius expressions were extended by two multiplying terms (see Eq. 2) as suggested by Wang and coauthors [10] in their underlying kinetic study on biomass, resulting in the next general form:

$k_{\mathrm{r}}=\frac{\mathrm{d} x_{\mathrm{int}}}{\mathrm{d} t}=\sum_{i=1}^{p} c_{\mathrm{i}} A_{\mathrm{i}} \mathrm{e}^{-\mathrm{E}_{\mathrm{i}} /(\mathrm{RT})}(1-x)^{\mathrm{n}_{\mathrm{i}}}\left(x+z_{\mathrm{i}}\right)^{\mathrm{m}_{\mathrm{i}}}$.

To get the parameters of Eq. (7), an optimum-seeking procedure was applied based on the least squares criterion. (Note that this procedure results in the product $c_{\mathrm{i}} A_{\mathrm{i}}$. It should be separated to its two terms on condition that these parameters with physical meanings are of interest. In this case, $c_{\mathrm{i}}$, the mass ratio of the given fraction should be read from the TG diagram.)

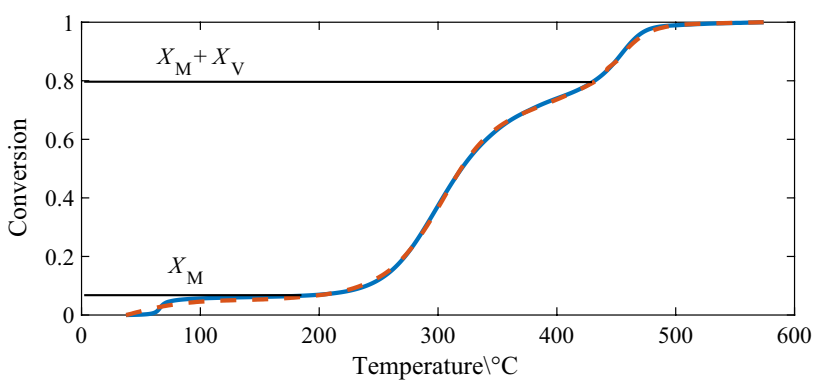

Fig. 1 Intrinsic conversion curve gained directly from the TG measurement of the investigated fuel (blue -). The result of curve fitting to be discussed in the actual section is also shown (red - -). As part of the fuel characterization, the values of $X_{\mathrm{M}}$ and $X_{\mathrm{V}}$ can be read from the diagram as shown. (Color figure online) 
Table 2 Proximate data of the sample

\begin{tabular}{|c|c|c|c|c|c|c|}
\hline $\begin{array}{l}\text { Reference: } \\
\text { Index extension: }\end{array}$ & & & $\begin{array}{l}\text { Ash-free } \\
-\end{array}$ & $\begin{array}{l}\text { Dry } \\
\mathrm{d}\end{array}$ & $\begin{array}{l}\text { Dry, ash-free } \\
\text { daf }\end{array}$ & $\begin{array}{l}\text { As received } \\
\text { ar }\end{array}$ \\
\hline Moisture & $X_{\mathrm{M}}$ & mass $\%$ & 5.31 & - & - & 4.98 \\
\hline Volatile & $X_{\mathrm{V}}$ & mass $\%$ & 76.19 & 75.19 & 80.46 & 71.44 \\
\hline Ash & $X_{\mathrm{A}}$ & mass $\%$ & - & 6.59 & - & 6.23 \\
\hline
\end{tabular}

Values in the ash-free column are used for the calculations without any further index extensions. The $X_{\mathrm{M}}$ and $X_{\mathrm{V}}$ values are taken from Fig. 1, while $X_{\mathrm{A}}$ from its original, still not normalized version in the same way

\section{Planning and carrying out the macroscopic measurement}

In the current method, macroscopic measurement should be taken. Generally speaking, any experimental setup could be applied; however, some characteristics of the method should be considered while planning the experiment. As the 3D model of the whole experimental volume must be set up (to be introduced in detail in the upcoming two subsections), it is preferable to apply well-defined and easy-to-model circumstances. Further, because the simultaneous effects of char combustion and diffusion must be distinguished (see Eq. (4), bottom line), their keeping in the same order of magnitude at least during some parts of the experimental time must also be assured and checked at the end. The variables concerned are $\eta_{\mathrm{m}} k_{\mathrm{r}}$ and $k_{\mathrm{d}}$, and they were very close to each other both at the initial phase of the char combustion and at the very end of it. (Note that if definite diffusion controls throughout the entire char combustion process in all experimental and modeled states, $\eta_{\mathrm{m}} k_{\mathrm{r}}$ can be omitted from the bottom line of Eq. (4). However, it was kept in the current study for general applicability.)

\section{Calculating the particle temperature profile}

For evaluating the macroscopic measurements, it is essential to know the temperature history of the particles throughout the investigated combustion process. Moreover, because of the significant spatial extent and irregular shape of the particles, their internal inhomogeneous temperature must also be considered.

Although the sizes of the investigated, industrially relevant particles are rather big compared to others used in the pulverized coal combustion, e.g., they are far too small for applying several reliably fixed thermocouples on them for the experiment duration in most cases. Further, analytically handling thermal inhomogeneities cannot be done in most cases. However, if the experimental setup is simple enough, and the side conditions are well defined as well, CFD modeling can beneficially be applied. This is the method we propose to describe the particle temperature throughout the experimental combustion process.

\section{Calculating the gas mass transfer around the particle}

Gas transfer around the particle is another phenomenon to be considered and described to get the missing element of the model. A description based on the Sherwood number $S h$ is the commonly used method [26] because a big number of theoretical and empirical correlations are available for both calculating the $S h$ number and calculating $k_{\mathrm{d}}$ from it. The major problem is that most of the above correlations are valid explicitly for spherical particles. This is the reason why another way has to be chosen for the nonspherical particles.

The 3D CFD model discussed above can also be used for this purpose, but it must be extended also to describe the fluid dynamical behavior of the entire experimental volume. Boundary conditions must also be set properly. This calculation can be carried out, and from its very detailed 3D oxygen mass transfer data, their overall $k_{\mathrm{d}}$ function can be gained by means of an integration over the particle surface.

\section{Calculating the parameters of the mass-based reaction effectiveness factor}

After carrying out the procedure described in "Biofuel particles and their intrinsic kinetic characterization" section through "Calculating the gas mass transfer around the particle" section, the only unknown in Eq. (4) remains $\eta_{\mathrm{m}}$; thus, it can be already determined. The proposed form for this function is (5)-(6); hence, the goal is to find all the free parameters of these equations fitting the data gained in the previous steps best. Several optimum-seeking procedures are available and can be applied, among which the genetic algorithms offer significant advantages, and that is why they were used for the example of biofuel particles.

The basic principles of the genetic algorithms are originated from the Darwinian evolution theory, as summarized by McCall [27]. It works by generating a set of solutions for the same problem in every generation, for which a set of parameters is randomized every time. These solutions are compared to a desired value, and the best parameter sets are selected to calculate the next generation, until an error 
criterion is reached. This error is called the fitness value $F$, defined by the fitness function.

The most commonly applied criteria are based on the least squares, but they also consider the total number of discrete data points, $N_{\tau}$, applied for comparison throughout the discrete time $\tau$, to make different cases easier to compare, as follows:

$F=\frac{\sum_{\tau}\left(x_{\text {app,meas }}(\tau)-x_{\text {app,calc }}(\tau)\right)^{2}}{N_{\tau}}$.

Here $x_{\text {app,meas }}(\tau)$ and $x_{\text {app,calc }}(\tau)$ are the measured and calculated values of the apparent conversion in the actual case.

\section{Results and discussion}

Sunflower seed shell pellets as renewable fuel particles were used to prove the procedure proposed and described in the previous section (No. "Materials and methods" section, Mat. and Methods). The procedure and the resulted data on each of its step for this specific test fuel will be introduced in this section; the structure is identical to that of the previous one to identify and follow it easily. The fuel itself, together with its initial characteristics gained before the current procedure, was already introduced, in an appropriate subsection (No. "Biofuel particles and their intrinsic kinetic characterization" section).

\section{Intrinsic kinetic data}

The resulted parameters of the curve fitting to the measured TG curve of the investigated fuel are summarized in Table 3, and the curve itself is shown in Fig. 1 by red dashed line. The number of the superpositioning components is set to $p=3$, as visible. This is a satisfactory choice in $\operatorname{good}$ accordance with the results of some earlier investigations using the same method $[13,14]$.

The activation energies are a little lower than the ones presented by the above-mentioned works, but it resonates well with the quite short conversion curve (it is finished before $600{ }^{\circ} \mathrm{C}$ instead of $800{ }^{\circ} \mathrm{C}$, which is the usual approximate final temperature in case of most biomasses).

\section{Macroscopic measurement}

An electrically heated oven equipped with a digital balance for measuring continuously the mass loss of the sample particles was used in the example measurement, as introduced in detail elsewhere [28]. The internal arrangement of the oven was kept as simple as possible; hence, the next step of the procedure requires its geometrical modeling. The internal dimensions of the heated volume are $445 \mathrm{~mm} \times 170 \mathrm{~mm}$ $\times 131 \mathrm{~mm}(\mathrm{~L} \times \mathrm{W} \times \mathrm{H})$. At the beginning of each measurement, the initial oven temperature was set to $850^{\circ} \mathrm{C}$, and the samples were put on the sample holder. Fourteen pellets were used in the measurements arranged next to each other on the spoon. The sample was not prepared in any way, as the aim was to stay as close as possible to the conditions of industrial usage, and the robust measuring equipment did not demand it either. The sample holder was connected to a digital scale, and they were fixed on a small wheeled cart, which made it possible to push the sample in without disturbing the initial few seconds of the measurement. After the insertion, the entrance of the oven was covered with isolation material having a large enough hole to provide satisfactory ventilation.

The result of this measurement on the example fuel particles is shown in Fig. 2 (continuous line).

\section{The particle temperature profile}

The transient numerical model of the experimental layout was developed in a commercial CFD code (COMSOL 5.3).

$850^{\circ} \mathrm{C}$ was set as initial temperature of the air inside the oven and its walls, and $20^{\circ} \mathrm{C}$ of the sample. The heat released by the flame was modeled as a heat source in a

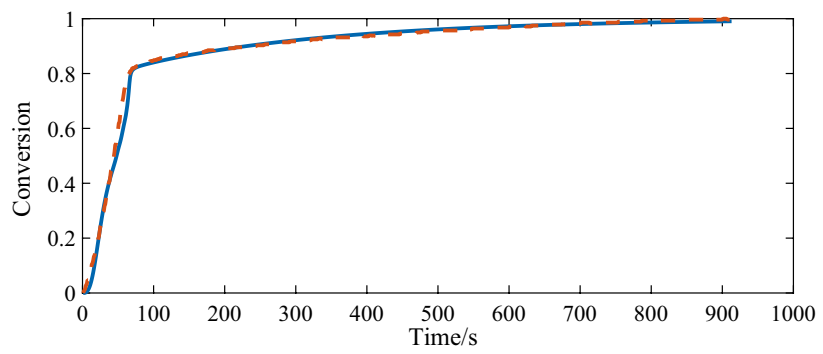

Fig. 2 Measured (blue -) and calculated (red - -) apparent conversion rate throughout the entire combustion process. (Color figure online)
Table 3 The best-fitting intrinsic parameters

\begin{tabular}{lllllll}
\hline$i$ & $c_{\mathrm{i}}$ & $A_{\mathrm{i}} / \mathrm{s}^{-1}$ & $E_{\mathrm{i}} / \mathrm{J} \mathrm{mol}^{-1}$ & $z_{\mathrm{i}}$ & $m_{\mathrm{i}}$ & $n_{\mathrm{i}}$ \\
\hline 1 & 0.07 & $2.27 \times 10^{4}$ & $2.44 \times 10^{4}$ & 0.060 & 3.10 & 5.350 \\
2 & 0.73 & $6.66 \times 10^{4}$ & $7.20 \times 10^{4}$ & 0.025 & 0.50 & 2.410 \\
3 & 0.20 & $1.68 \times 10^{5}$ & $9.41 \times 10^{4}$ & 0.042 & 1.03 & 1.013 \\
\hline
\end{tabular}


Fig. 3 Discretization of a sample for numerical simulation of its temperature inhomogeneity

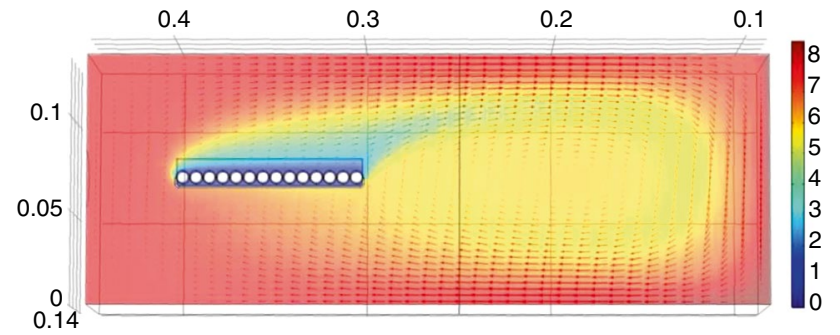

Fig. 4 CFD modeling of the apparent experimental space. (The color code is for the oxygen concentration in $\mathrm{mol} \mathrm{m}^{-3}$, the arrows show the directions and relative magnitudes of gas flow. All sizes in m.). (Color figure online)

volume above the sample, and its magnitude was set according to the measured volatile release. In a similar way, this volatile release was considered as a significant factor influencing also the fluid mechanical environment around the particle (although this effect is negligible in many cases, but not with biofuels characterized by very high volatile content).

It was assumed in the model that the temperature in the flame was high enough to burn the volatile completely. Heat convection, conduction, and radiation were considered in the calculation, the latter one by the surface-to-surface method for the solids, and by the discrete ordinates method for the flame.

To describe the inhomogeneous warming-up of the particle during the experiment, the cylindrical samples were discretized into 15 parts, as shown in Fig. 3. A characteristic temperature in the center point of each part was logged throughout the simulated warming-up procedure.

The distribution of the parts is very sample dependent, and it should be designed specifically for every fuel particle based on its geometry. It should be fine enough to precisely describe the interior heat transfer, but not finer than what is reasonably suggested by the mesh of the numerical method.

\section{The gas mass transfer around the particle}

Because of the marked limitations of the generally applied method based on the Sherwood number (see "Calculating the gas mass transfer around the particle" section), another way had to be chosen for the investigated biofuel particles. The previously introduced 3D CFD model was extended to the entire experimental space. This volume, together with
Table 4 Parameters of the mass-related kinetic effectiveness factor $\eta_{\mathrm{m}}$ (Eq. 5) as results of the optimum-seeking procedure

\begin{tabular}{|c|c|c|c|c|}
\hline Parameter: & $A_{\eta}$ & $E_{\eta}$ & $m_{\eta}$ & $\eta_{\mathrm{m}, 0}$ \\
\hline Unit: & - & - & - & - \\
\hline Value: & $1.20 \times 10^{5}$ & 6.20 & 0.720 & $2.44 \times 10^{-3}$ \\
\hline
\end{tabular}

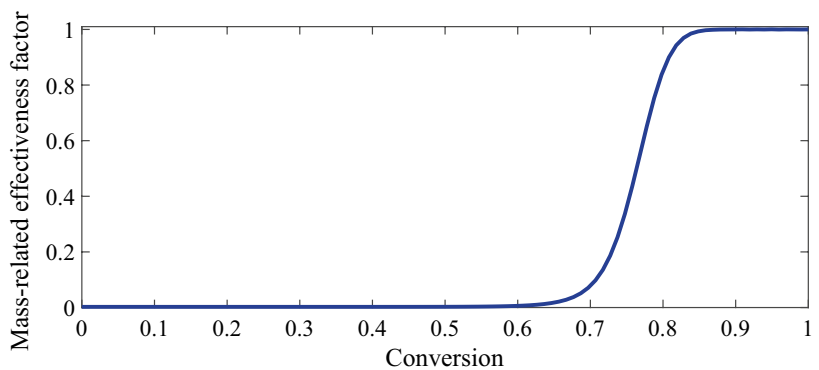

Fig. 5 Graphical representation of $\eta_{\mathrm{m}}(x)$ after solving (5)-(6) numerically

the most important calculated results, can be seen in Fig. 4 for the actual case.

\section{The mass-related reaction effectiveness factor}

To find the free parameters of (5)-(6), the direct fitting method was used. A MATLAB ${ }^{\circledR}$ code was developed, which called the built-in genetic algorithm provided by Matlab's Optimization Toolbox.

In the actual case, for simplicity, and because of its negligible effect on the accuracy of the result, a constant value of $\varepsilon=1 \mathrm{E}-4$ was used. Besides the above favorable effects of this decision, it also resulted in a marked decrease in the computational demand of the optimum-seeking procedure; hence, it can be advised for most other cases as well.

The best fit could be achieved by the parameters in Table 4, and the resulted shape of the function is shown in Fig. 5 graphically.

Note that instead of the direct fitting method described above, a more traditional procedure could also be chosen. In this way, optimal $\eta_{\mathrm{m}}$ values should be searched at several points along the range of the conversion rate $x$, and the final, explicit form of $\eta_{\mathrm{m}}$ could be the result of a simple function fitting to these points. This method was also checked, but it was found that it underperformed the proposed one. The reason is that in spite of the very flat regions around the optimum points of the first fitting step, the second functionfitting step tries to adhere too strictly to those points. However, this procedure (more precisely, its first step) can be a useful tool for getting an initial impression on the possible shape of $\eta_{\mathrm{m}}$ for the specific fuel actually concerned. Further, this first, preliminary step was done for the example of fuel 
of this study, and it justified our a priori thought about the shape of $\eta_{\mathrm{m}}(x)$ as it converges to unity at $x \rightarrow 1$. The practical meaning of this characteristic is that, at the very end of the char combustion process, the particle is very small, that is, it behaves similarly to the very small sample in the TG apparatus.

\section{Verification}

By this point, $\eta_{\mathrm{m}}$, the bridging element between intrinsic and apparent descriptions was determined by a generally applicable method. Thus, the apparent model is available, and now its verification must be done. Several levels (or strengths) of verification can be selected. In this study, a generally applied, mid-strength method was followed as the calculated mass conversion $\left(x_{\text {app }}\right)$ data were compared to their measured values.

A comparison between measurement and model prediction is shown in Fig. 2. Note that the calculated $x$ values in this figure were taken from the pure use of the model as described in equations (4)-(5) with the parameters of Table 4.

\section{Conclusions}

In this work, a complex model was developed to connect the theoretical results coming from laboratory-scale (intrinsic) reaction kinetics and the (apparent) combustion of real, macro-sized and special-shaped solid fuels as a function of conversion. The method starts with a macroscopic mass change measurement on high temperature in an easily modelable environment, which results in an $x(t)$ conversion graph. It has to be substituted into a modified Arrhenius equation, in which all other variables can be gained by a TG measurement and numerical simulations. So, finally, the only unknown variable, the mass-related reaction effectiveness factor, $\eta_{\mathrm{m}}$, can be calculated. This function is the key for modeling the entire combustion process of the same particles under different specific circumstances and could be used as a handy theoretical tool to describe this complex process relatively simply.

For setting up and testing the prototype of the model, sunflower seed shell pellets were chosen, and the results of the verification on this biofuel example were convincing. The presented experimental results were processed by the discussed technique, and the $\eta_{\mathrm{m}}$ function was created, which is now available to be used in more complex models concerning real practical problems.

In case of applying the method for other biomasses, it should be considered that every biomass behaves differently because of different intrinsic kinetics and geometry. If the sample is small, it is not advised to use this technique as it will just make the calculation more complex without any benefit. However, in case of bigger particles where the internal heat transfer is relevant and the reaction front is moving inside from the edges, using the mass-related reaction effectiveness factor will significantly increase the precision. To achieve the best results, every parameter should be reconsidered and recalculated based on new numerical simulations and new measurements for the new sample. It is also possible that an entirely new form is needed for $\eta_{\mathrm{m}}$; however, the one suggested in the paper is quite complex and general to be used as at least a starting point for all biomasses.

Acknowledgements Open access funding provided by Budapest University of Technology and Economics (BME). This work was supported by the National Research, Development and Innovation Fund of Hungary in the frame of FIEK_16-1-2016-0007 (Higher Education and Industrial Cooperation Center) project.

The research reported in this paper has been supported by the National Research, Development and Innovation Fund (TUDFO/51757/2019-ITM, Thematic Excellence Program).

Open Access This article is distributed under the terms of the Creative Commons Attribution 4.0 International License (http://creativeco mmons.org/licenses/by/4.0/), which permits unrestricted use, distribution, and reproduction in any medium, provided you give appropriate credit to the original author(s) and the source, provide a link to the Creative Commons license, and indicate if changes were made.

\section{References}

1. Jiang X, Chen D, Ma Z, Yan J. Models for the combustion of single solid fuel particles in fluidized beds: a review. Renew Sustain Energy Rev. 2017;68(Part 1):410-31.

2. Laurendeau NM. Heterogeneous kinetics of coal char gasification and combustion. Prog Energy Combust Sci. 1978;4:221-70.

3. Gomez-Barea A, Leckner B. Modeling of biomass gasification in fluidized bed. Prog Energy Combust Sci. 2010;36:444-509.

4. Khodaei H, Al-Abdeli YM, Guzzomi F, Yeoh GH. An overview of processes and considerations in the modelling of fixed-bed biomass combustion. Energy. 2015;88:946-72.

5. Di Blasi C. Modeling chemical and physical processes of wood and biomass pyrolysis. Prog Energy Combust Sci. 2008;34:47-90 WOS:000257203400002.

6. Field MA, Gill DW, Morgan BB, Hawksley PGW. Combustion of pulverised coal. Leatherhead (Sy.): B.C.U.R.A., British Coal Utilisation Research association ed., 1967; OCLC: 461592.

7. Field M. Rate of combustion of size-graded fractions of char from a low-rank coal between $1200 \mathrm{~K}$ and $2000 \mathrm{~K}$. Combust Flame. 1969;13:237-52.

8. Levenspiel O. Chemical reaction engineering. 3rd ed. New York: Wiley; 1998.

9. Di Blasi C. Combustion and gasification rates of lignocellulosic chars. Prog Energy Combust Sci. 2009;35(2):121-40.

10. Wang L, Varhegyi G, Skreiberg O, Li T, Gronli M, Antal MJ. Combustion characteristics of biomass charcoals produced at different carbonization conditions: a kinetic study. Energy Fuels. 2016;30:3186-97. 
11. Aboulkas A, El Harfi K, El Bouadili A. Thermal degradation behaviors of polyethylene and polypropylene. Part I: pyrolysis kinetics and mechanisms. Energy Convers Manag. 2010;51:1363-9.

12. Varhegyi G, Szabo P, Jakab E, Till F, Richard J-R. Mathematical modeling of char reactivity in $\mathrm{Ar}-\mathrm{O}_{2}$ and $\mathrm{CO}_{2}-\mathrm{O}_{2}$ mixtures. Energy Fuels. 1996;10:1208-14.

13. Conesa JA, Rey L. Thermogravimetric and kinetic analysis of the decomposition of solid recovered fuel from municipal solid waste. J Therm Anal Calorim. 2015;120:1233-40.

14. Galvagno S, Casu S, Martino M, Di Palma E, Portofino S. Thermal and kinetic study of tyre waste pyrolysis via TG-FTIR-MS analysis. J Therm Anal Calorim. 2007;88:507-14.

15. Agraniotis M, Nikolopoulos N, Nikolopoulos A, Grammelis P, Kakaras E. Numerical investigation of solid recovered fuels' co-firing with brown coal in large scale boilers-evaluation of different co-combustion modes. Fuel. 2010;89:3693-709 WOS:000282086200012.

16. Momeni M, Yin C, Kær SK, Hansen TB, Jensen PA, Glarborg P. Experimental study on effects of particle shape and operating conditions on combustion characteristics of single biomass particles. Energy Fuels. 2013;27:507-14

17. Bibrzycki J, Mancini M, Szlek A, Weber R. A char combustion sub-model for CFD-predictions of fluidized bed combustion-experiments and mathematical modeling. Combust Flame. 2016;163:188-201.

18. Schiemann M, Haarmann S, Vorobiev N. Char burning kinetics from imaging pyrometry: particle shape effects. Fuel. 2014;134:53-62.

19. Liu Y, Fu P, Zhang B, Yue F, Zhou H, Zheng C. Study on the surface active reactivity of coal char conversion in $\mathrm{O}_{2} / \mathrm{CO}_{2}$ and $\mathrm{O}_{2} / \mathrm{N}_{2}$ atmospheres. Fuel. 2016;181:1244-56.

20. Pereira $\mathrm{C}$, Pinho C. Influence of particle fragmentation and nonsphericity on the determination of diffusive and kinetic fluidized bed biochar combustion data. Fuel. 2014;131:77-88.
21. Johansen JM, Gadsbøll R, Thomsen J, Jensen PA, Glarborg P, Ek P, De Martini N, Mancini M, Weber R, Mitchell RE. Devolatilization kinetics of woody biomass at short residence times and high heating rates and peak temperatures. Appl Energy. 2016;162:245-56.

22. Scala F. Mass transfer around active particles in fluidized beds. In: El-Amin M, editor. Mass transfer in multiphase systems and its applications. London: InTech; 2011.

23. Li J, Zhang J. A theoretical study on char combustion of ellipsoidal particles. Combust Sci Technol. 2016;188:40-54.

24. Szentannai P, Szucs B. Vertical arrangement of SRF particles in a stationary fluidized bed. Powder Technol. 2018;325:209-17.

25. Bakos LP, Mensah J, Laszlo K, Igricz T, Szilagyi IM. Preparation and characterization of a nitrogen-doped mesoporous carbon aerogel and its polymer precursor. J Therm Anal Calorim. 2018;134:933-9.

26. Scala F, Solimene R, Montagnaro F. 7-Conversion of solid fuels and sorbents in fluidized bed combustion and gasification. In: Scala F, editor. Fluidized bed technologies for near-zero emission combustion and gasification, Woodhead Publishing Series in energy. Sawston: Woodhead Publishing; 2013. p. 319-87.

27. McCall J. Genetic algorithms for modelling and optimisation. J Comput Appl Math. 2005;184:205-22.

28. Szentannai P, Bozi J, Jakab E, Osz J, Szucs T. Towards the thermal utilisation of non-tyre rubbers-Macroscopic and chemical changes while approaching the process temperature. Fuel. 2015;156:148-57.

Publisher's Note Springer Nature remains neutral with regard to jurisdictional claims in published maps and institutional affiliations. 